\title{
A Method for Monitoring Intensity During Aquatic Resistance Exercises
}

\author{
Juan C. Colado, ${ }^{1}$ Victor Tella, ${ }^{\mathbf{1}}$ and N. Travis Triplett ${ }^{\mathbf{2 , 3}}$ \\ ${ }^{1}$ Department of Physical Education and Sports, University of Valencia, Valencia, Spain; ${ }^{2}$ Neuromuscular Laboratory, \\ Department of Health, Leisure and Exercise Science, Appalachian State University, Boone, North Carolina; \\ ${ }^{3}$ Invited Researcher, University of Valencia, Valencia, Spain
}

\begin{abstract}
Colado, JC, Tella, V, and Triplett, NT. A method for monitoring intensity during aquatic resistance exercises. $J$ Strength Cond Res 22(6): 2045-2049, 2008-The aims of this study were (i) to check whether monitoring of both the rhythm of execution and the perceived effort is a valid tool for reproducing the same intensity of effort in different sets of the same aquatic resistance exercise (ARE) and (ii) to assess whether this method allows the ARE to be put at the same intensity level as its equivalent carried out on dry land. Four healthy trained young men performed horizontal shoulder abduction and adduction (HSAb/Ad) movements in water and on dry land. Muscle activation was recorded using surface electromyography of 1 stabilizer and several agonist muscles. Before the final tests, the ARE movement cadence was established individually following a rhythmic digitalized sequence of beats to define the alternate $\mathrm{HSAb} / \mathrm{Ad}$ movements. This cadence allowed the subject to perform 15 repetitions at a perceived exertion of 9-10 using Hydro-Tone Bells. After that, each subject performed 2 nonconsecutive ARE sets. The dry land exercises (1 set of $\mathrm{HSAb}$ and 1 set of HSAd) were performed using a dual adjustable pulley cable motion machine, with the previous selection of weights that allowed the same movement cadence to be maintained and the completion of the same repetitions in each of the sets as with the ARE. The average normalized data were compared for the exercises in order to determine possible differences in muscle activity. The results show the validity of this method for reproducing the intensity of effort in different sets of the same ARE, but is not valid for matching the same intensity level as kinematically similar land-based exercises.
\end{abstract}

KEY WoRDS electromyography, drag force, movement cadence, trunk stabilization

Address correspondence to Juan C. Colado, juan.colado@uv.es. 22(6)/2045-2049

Journal of Strength and Conditioning Research

(c) 2008 National Strength and Conditioning Association

\section{INTRODUCTION}

A quatic resistance exercises (AREs) are those exercises performed in water using special devices that take advantage of the increased drag force and are similar to their counterparts on dry land. These exercises are commonly used in muscle rehabilitation programs $(13,14)$. However, the lack of methodological criteria to produce consistent resistance when performing these types of exercises has limited their use in the fitness field (10). This is despite the evidence obtained from the work of Takeshima et al. (13) and Pöyhönen et al. (11), which highlight the effectiveness of AREs in achieving both neuromuscular and structural adaptations. Since these investigations used sample populations of individuals who were undergoing rehabilitation or had little experience with intensive strength training, it is likely that AREs are still thought to be for compromised populations and are not used more in strength training programs for the average individual or an athlete.

Therefore, the aims of the current study were as follows: (i) to check whether monitoring cadence and technique allowed us to reproduce the intensity of muscle activation in different sets of the same ARE; (ii) to evaluate whether AREs using devices that increase drag force can generate an adequate threshold of muscle activation in the pectoral, upper dorsal, and lumbar regions of fit individuals who are used to strength exercises; and (iii) to compare the muscle activation achieved in AREs with that obtained in kinematically similar dry land resistance exercises.

\section{Methods}

\section{Experimental Approach to the Problem}

Surface electromyography during the performance of shoulder horizontal adduction and abduction was performed in the agonist muscles of those movements (pectoralis major, posterior deltoid) and in one trunk stabilizer muscle (erector spinae lumborum). The movement cadence and the size and position of the equipment were monitored (3) in an attempt to objectively control exercise intensity. Muscle activity was determined in shoulder adduction and abduction on a horizontal plane. The range of motion was standardized such that during horizontal shoulder adduction the subject's hands nearly touched, with shoulder-level abduction to 
$80^{\circ}$ and $\mathrm{a} 20^{\circ}$ elbow bend without assistance from the musculature of the remaining body segments (Figures 1 and 2).

Both water- and land-based versions of these movements were performed standing without external points of support. In water, the movements were performed with Hydro-Tone Bells (Aquatic Fitness Systems, Inc., Huntington Beach, CA) (15) in the vertical position, and on dry land, the movements were performed with a dual adjustable pulley cable motion machine. Since in water these movements are concentric only, on land, the muscular activation of only the concentric phase of each movement was assessed. Despite being familiar with the movement involved with both devices, the subjects received instruction before performing the exercise and feedback was provided in the correct execution of the movements during the tests.

\section{Subjects}

Four fit young men with low subcutaneous fat levels with no orthopedic limitations participated in this study. Subject characteristics were as follows: age (mean $\pm S D$ ), $24.33 \pm$ 0.58 years; body mass, $80.27 \pm 13.04 \mathrm{~kg}$; body fat, $12.03 \pm$ $2.22 \%$. Body fat was analyzed using bioelectrical impedance (Tanita body composition analyzer, model TBF-418 MA). All subjects had previous experience in performing strength exercises in water. In addition, the subjects had a minimum of 2 years experience with normal (dry land) resistance exercises, with an average weekly frequency of $3.7 \pm 1.1$ sessions. The study was approved by the Institutional Review Board at the Catholic University of Murcia (Spain), and all subjects provided written informed consent before taking part in the study.

\section{Exercise Protocol}

After a standardized 5-minute warm-up, the appropriate cadence of movement was identified for each subject in the water that allowed a set of $15 \pm 2$ repetitions at or near

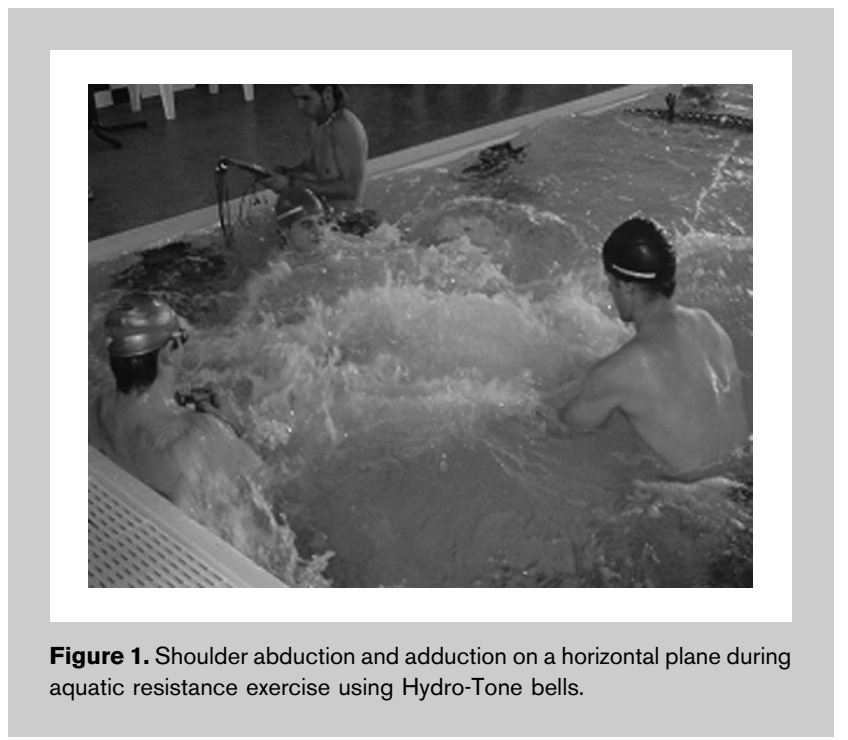

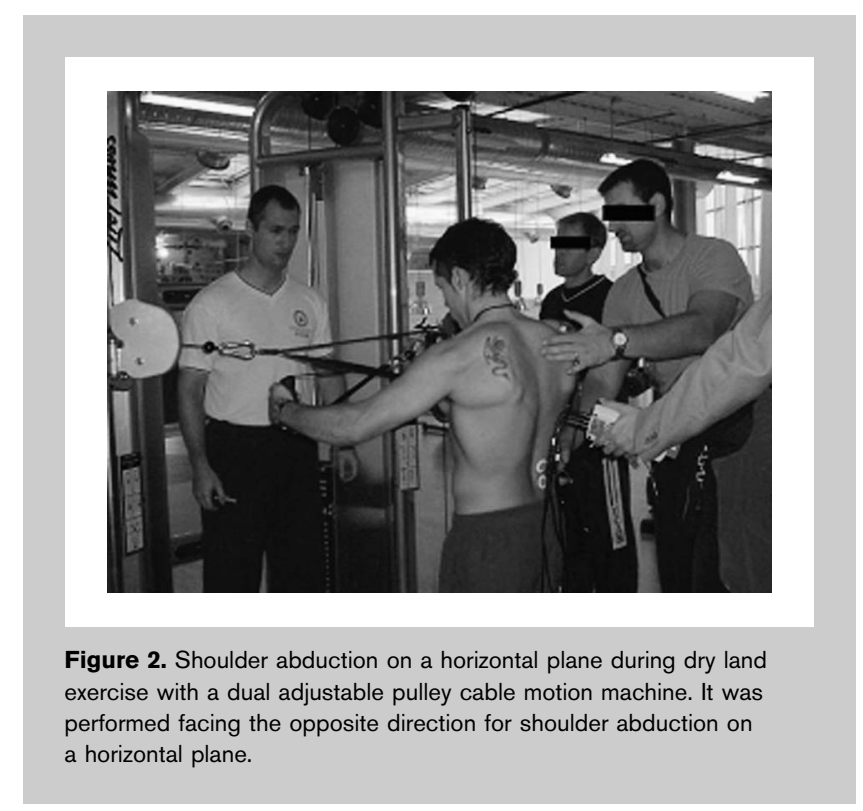

maximum effort, while maintaining correct technique. Optimum cadence was determined by trial-and-error selection of different cadences (emitted by an acoustic metronome), with a 5-minute rest between, until the appropriate cadence was found.

Subjects rested for at least 30 minutes and performed the same warm-up as before and then resistance was determined. The horizontal shoulder adduction movement (dry land) was performed using a dual adjustable pulley cable motion machine. The initial resistance was set where it was thought the desired number of repetitions could be achieved at the cadence chosen for the water version of the exercise. As before, each subject was allowed to attempt different weights, with 5-minute rest between, until the target number of repetitions at cadence was matched, which indicated the correct resistance for the later dynamic exercise set. The same protocol was then followed with the horizontal shoulder abduction movement.

After at least a 20-minute rest and performing the same warm-up, each subject carried out the horizontal shoulder adduction and abduction maximum voluntary isometric contraction (MVIC) tests for muscle peak muscle activity according to established protocols (12) and using a dual adjustable pulley cable machine. Subjects also performed an MVIC for peak muscle activity for the erector spinae lumborum on a Roman chair. There was a minimum rest period of 10 minutes between the 3 tests.

The exercise protocol began after a minimum of 10 -minute rest period and a repeat of the 5-minute warm-up, then 1 set of each of the horizontal shoulder adduction and abduction movements on the cable machine was performed at the cadence and resistance previously determined. After repeating the specified warm-up, each subject carried out one set of aquatic resistance exercise of horizontal shoulder

2046

Journal of Strength and Conditioning Research"

Copyright $($ National Strength and Conditioning Association. Unauthorized reproduction of this article is prohibited. 
ad-abduction using the Hydro-Tone Bells at the previously defined movement cadence. After a minimum 5-minute rest period, the subjects repeated the aquatic exercise while receiving additional resistance of 30 water currents per minute with a force of $68.55 \mathrm{~N}$ from the front (Figure 1).

\section{Electromyography}

Muscle activity was determined using surface electromyography (model ME6000, Mega Electronics, Kuppio, Finland). Bipolar surface electrodes were applied $(\mathrm{Ag}-\mathrm{AgCl})$ with conducting gel (M-00-S; Medicotest, Olstikke, Denmark) and used to measure signals from the chosen muscles $(6,8)$. For the aquatic measurements, a kit for aquatic use was employed consisting of a waterproof cover with integrated electromyographic electrodes (Figure 1). The electromyographic electrodes were protected by a waterproof film during the water exercises (4). All data collected were analyzed with the software provided by the manufacturer (Mega Win v.2.3).

\section{Statistical Analyses}

The data were analyzed using the SPSS program. Standard procedures were used to calculate means and SDs. Student's $t$ test with 1 degree of freedom was performed on the normalized integrated electromyographic values to identify any significant differences between the means for the muscle activity of each of the muscles evaluated in each of the exercises carried out. Statistical significance was accepted at $p \leq 0.05$.

\section{Results}

Table 1 shows that the mean muscle activation achieved by the pectoralis major muscle was always significantly lower than that obtained for the posterior deltoid muscle, regardless of the exercise medium. The data also showed that there was significantly greater activation of the muscles evaluated when the exercises were carried out in water compared with when they were carried out on dry land (Table 1 and Figure 3D). There were no statistically significant differences in mean muscle activation in the agonist muscles (Figure $3 \mathrm{~A}$ and $\mathrm{B}$ ) in the various conditions, although 1-2 subjects showed a significant difference between the sets of the 2 water conditions. Conversely, there was a significant difference in erector spinae lumborum activation in the sets of the 2 water conditions (Figure $3 \mathrm{C}$ and Table 1).

\section{Discussion}

The degree of muscle activation measured during strength exercises using integrated electromyography is linked to the level of intensity with which an exercise is performed and is an excellent indicator of the internal force generated and is positively correlated with force output, especially when evaluating concentric muscle actions (5). If it is assumed that, for a determined resistance, a higher cadence of movement leads to greater muscle activation, then maintaining that cadence should mean that muscle activation does not vary and that the intensity of the exercise has remained stable. As previously mentioned, monitoring intensity in aquatic exercises for strength training has been regarded as one of the main difficulties in prescribing this type of training, although several authors have partially approached this problem $(11,16)$. This investigation attempted to determine the intensity of aquatic exercise through muscle activation, controlling for speed of movement, surface area of aquatic equipment used, and body segment position. Since the same level of muscle activation when performing different sets of a specific exercise is related to the same intensity for the effort involved, as seems to have happened with the muscle activation achieved in this study, based on this preliminary evidence, this method could be regarded as valid for monitoring the intensity of AREs at different times and for different subjects.

Interestingly, the mean activation achieved for the pectoralis major muscle was always significantly less $(p \leq$ $0.05)$ than that obtained for the posterior deltoid muscle. The possible explanation for this difference could be that the pull musculature of these subjects was weaker than their press musculature, specifically $11.34 \pm 1.02 \%$. According to Baker and Newton (1) this difference could reach $15 \%$ for trained individuals. Therefore, this imbalance in strength limits the stronger muscle group, as it is limited by the cadence of movement that can be followed by the weaker muscle group.

TABLE 1. Means and SDs of the percentage of maximal voluntary isometric contraction for each muscle group in the different exercises evaluated.

\begin{tabular}{|c|c|c|c|c|}
\hline $\begin{array}{c}\text { Shoulder } \\
\text { movement type }\end{array}$ & $\begin{array}{l}\text { Evaluated } \\
\text { muscle }\end{array}$ & $\begin{array}{l}\text { Dry land(mean } \\
\quad \pm S D)\end{array}$ & $\begin{array}{c}\text { Aquatic } \\
\text { exercise }(\text { mean } \pm S D)\end{array}$ & $\begin{array}{l}\text { Aquatic exercise with } \\
\text { currents (mean } \pm S D)\end{array}$ \\
\hline Adduction & Pectoralis major & $43.21 \pm 27.64^{\mathrm{a}}$ & $49.24 \pm 28.56$ & $51.17 \pm 8.62^{\mathrm{a}}$ \\
\hline Abduction & Posterior deltoid & $60.85 \pm 16.80^{\mathrm{b}, \mathrm{c}}$ & $70.76 \pm 15.00^{\mathrm{b}}$ & $75.28 \pm 14.50^{c}$ \\
\hline Adduction & Erector spinae lumborum & $16.40 \pm 12.12^{\mathrm{d}, \mathrm{e}, \mathrm{f}}$ & $42.69 \pm 25.74^{\mathrm{e}, \mathrm{g}, \mathrm{i}}$ & $54.98 \pm 29.75^{\mathrm{f}, \mathrm{h}, \mathrm{i}}$ \\
\hline Abduction & Erector spinae lumborum & $24.31 \pm 9.66^{\mathrm{d}, \mathrm{g}, \mathrm{h}}$ & & \\
\hline
\end{tabular}

a,b,c,d,e,f,g,h,iSignificant differences between the pairs indicated $(p \leq 0.05)$. 

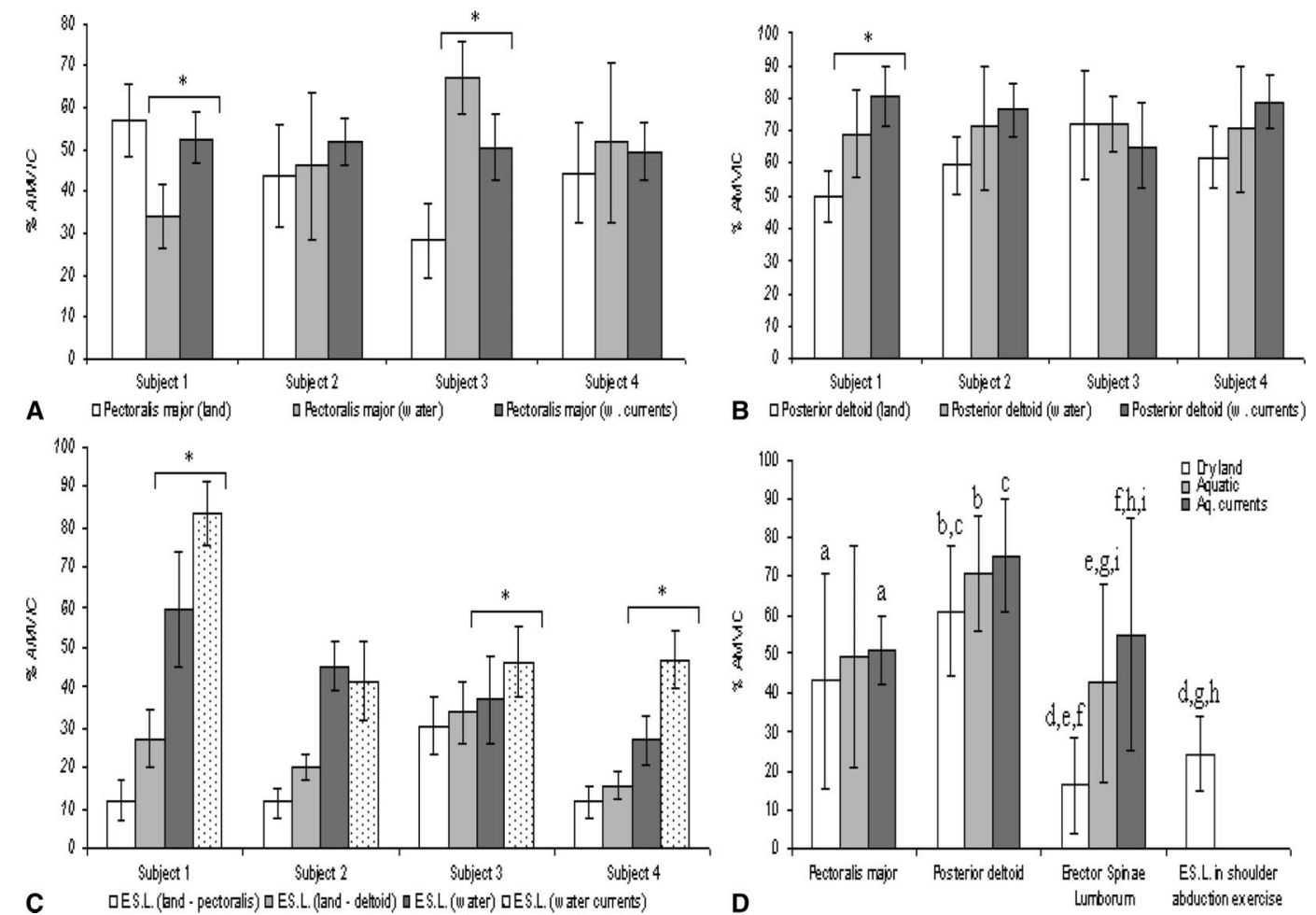

Figure 3. Average percentage of maximal voluntary isometric contraction (\%avgMVIC) for each subject in the different exercises evaluated for the pectoralis major $(A)$, posterior deltoid $(B)$, erector spinae lumborum (ESL) (C); each muscle group in the different exercises evaluated (D). "Significant difference between the muscle activation achieved in the first and second sets of the aquatic resistance exercise $(p \leq 0.05)$. ${ }^{a}, b, c, d, e, f, g, h, i$ Significant difference between the pairs indicated $(p \leq 0.05)$

This can also be seen in the high $S D$ for the muscle activity of the pectoralis major and could suggest that the resistance offered in certain repetitions was insufficient despite the fact that they were performed using the appropriate cadence of movement.

There is only one study comparing muscle activation while carrying out kinematically similar exercises in the aquatic and terrestrial media during shoulder abduction movement (7), although this study did not use any equipment to increase the area of the mobilized segment. That study concluded that electromyographic activity in the exercises performed while immersed was less than that when they were performed on dry land when the speed of movement was low, with the data also revealing that even with high speeds, the level of muscle activation achieved was less than the minimum threshold needed to obtain adaptations in healthy muscle groups. The results of the current study are not in agreement with the Kelly et al. (7) findings, probably because the equipment that was used in the current investigation had a large surface area (projected area of $581 \mathrm{~cm}^{2}$ ) and a high average movement cadence used by the subjects $\left(71 \pm 5.03 \mathrm{~b} \cdot \mathrm{min}^{-1}\right.$ or $106.63 \pm$ $\left.7.71^{\circ} \cdot \mathrm{s}^{-1}\right)$. As a result, the data gathered in this study suggest that the muscle activation achieved in a series of 15 repetitions with a maximum or near maximum nature of effort achieved muscle activation threshold values that would allow for muscle adaptations.

The concept of maintaining a steady cadence of movement and using it to try to find a resistance that allows a certain number of repetitions with a maximum or near maximum nature of effort to be carried out and could allow all sets to be equal in terms of the stress to the body was based on Newton's laws of force, mass, and acceleration. This relationship should have been true regardless of the medium (water or land). The muscle activation of the agonists was indeed similar between sets in performed in water. However, for both the pectoralis major and the posterior deltoid muscles, there was a significant difference between the muscle activation achieved with the AREs and the equivalent performed on land using a dual adjustable pulley cable motion machine. Although the cadence was the same for both modes of exercise and the dry land load was adjusted for the cadence and effort combined, there seem to be other factors that might have influenced the results. Thus, the protocol used to monitor the resistance offered by water in AREs does not seem to be valid to equalize the conditions of these exercises with those performed on land.

2048 Journal of Strength and Conditioning Research 
The data provided by this study suggest that when AREs are performed, muscle activation of the erector spinae lumborum is significantly greater than that achieved in dry land conditions. Another important fact shown by the data is that when the AREs are performed against water currents, the additional instability that is deliberately caused increases the activation even more. The wide $S D$ for activity of this muscle group while the AREs were being performed could suggest a variable muscle response in function of the degree of balance/imbalance that the subjects undergo over all the repetitions performed by the extremities in a particular set. This highlights the difficulty that body stabilization might undergo while AREs are being performed (2), highlighting the importance of maintaining correct spinal position so as to avoid injury, something normally recommended when training against resistance (9).

\section{Practical Applications}

One of the most important applications of this investigation is the need to use an acoustic or visual metronome with AREs to identify a cadence of movement that is used to complete a certain number of repetitions at maximum or near maximum effort. The number of repetitions should lie within the optimal range for the goals of the person exercising (strength, hypertrophy, or local muscular endurance), with the prescribed resistance provided by the AREs being defined as the resistance offered by the water to the movement, which can be graded according to the cadence with which the movements are carried out. Therefore, the same intensity can be achieved with an ARE as long as subsequent sets of the same ARE maintain the initial cadence of movement, the same equipment, and the same technique for executing them. Intensity can be easily varied in a quantifiable manner by modifying the cadence of movement. In addition, water currents directed at the participant can be used to increase the activation of the muscles of the trunk.

\section{ACKNowledgments}

Supported by PMAFI-PI-01/1C/04 from the Research Funds Program of the Catholic University San Antonio in Murcia (Spain).

\section{REFERENCES}

1. Baker, DG and Newton, RU. An analysis of the ratio and relationship between upper body pressing and pulling strength J Strength Cond Res 18: 594-598, 2004.

2. Colado, JC. Physical Conditioning in the Aquatic Way. Barcelona: Paidotribo, 2004

3. Colado, JC, Tella, V, and Llop, F. Response to resistance exercise performed in water vs. on land. Portuguese J Sport Sci 6(Suppl 2): 361-363, 2006

4. Fujisawa, H, Suenaga, N, and Minami, A. Electromyographic study during isometric exercise of the shoulder in head-out water immersion. J Shoulder Elbow Surg 7: 491-494, 1998.

5. Glass, SC and Armstrong, T. Electromyographical activity of the pectoralis muscle during incline and decline bench presses. J Strength Cond Res 11: 163-167, 1997.

6. Kelly, BT, Kadrmas, WR, Kirkendall, DT, and Speer, KP. Optimal normalization tests for shoulder muscle activation: an electromyographic study. J Orthop Res 14: 647-653, 1996.

7. Kelly, BT, Roskin, LA, Kirkendall, DT, and Speer, KP. Shoulder muscle activation during aquatic and dry land exercises in nonimpaired subjects. J Orthop Sports Phys Ther 30: 204-210, 2000.

8. Konrad, P, Schmitz, K, and Denner, A. Neuromuscular evaluation of trunk-training exercises. J Athl Training 36: 109-118. 2001.

9. Panjabi, MM. Clinical spinal instability and low back pain. J Electromyogr Kinesiol 13: 371-379, 2003.

10. Pöyhönen, T, Keskinen, KL, Kyröläinen, H, Hautala, A Savolainen, J, and Mälkiä, E. Neuromuscular function during therapeutic knee exercise under water and dry land. Arch Phys Med Rehabil 82: 1446-1452, 2001.

11. Pöyhönen T, Sipilä, S, Keskinen, KL, Hautala, A, Savolainen, J, and Mälkiä, E. Effects of aquatic resistance training on neuromuscular performance in healthy women. Med Sci Sports Exerc 34: 2103-2109, 2002.

12. Soderberg, GL and Knutson, LM. A guide for use and interpretation of kinesiologic electromyographic data. Phys Ther 80: 485-498, 2000.

13. Takeshima, N, Rogers, ME, Watanabe, E, Brechue, EW, Okada, A, Yamada, T, Islam, MM, and Hayano, J. Water-based exercise improves health-related aspects of fitness in older women. $\mathrm{Med} \mathrm{Sci}$ Sports Exerc 33: 544-551, 2002.

14. Thein, JM and Brody, LT. Aquatic-based rehabilitation and training for the elite athlete. J Orthop Sports Phys Ther 27: 32-41, 1998.

15. Thein, JM and Brody, LT. Aquatic-based rehabilitation and training for the shoulder. J Athl Train 35: 382-389, 2000.

16. Tsourlou, T, Benik, A, Dipla, K, Zafeiridis, A, and Kellis, S. The effects of a 24-week aquatic training program on muscular strength performance in healthy elderly women. J Strength Cond Res 20: 811-818, 2006 